\title{
Prevalence and correlates of depression in patients with epilepsy in Sri Lanka
}

\author{
Silumini De Silva ${ }^{1}$, Amila Isuru $^{2}$, Asiri Rodrigo ${ }^{3}$, Lalith Kuruppuarachchi ${ }^{3}$ \\ (Index words: depression, epilepsy, antiepileptics)
}

\begin{abstract}
Background: Depression is one of the most common psychiatric disorder in patients with epilepsy and it is often associated with poor quality of life, increased risk of suicide and poor seizure control, yet remains underdiagnosed and undertreated. The prevalence and associations for depression in patients with epilepsy vary between studies reflecting regional and cultural influences. Therefore, it is important to identify unique attributes within a community on this phenomenon This is the first study from Sri Lanka on the prevalence and correlates of depression in patients with epilepsy.

Method: We conducted this cross-sectional study at the Epilepsy clinic, Colombo North Teaching Hospital, Ragama. All consenting patients with a diagnosis of epilepsy followed up at the clinic, during study period, were enrolled. Symptoms of depression were screened with Beck Depression Inventory II and diagnosis was confirmed with a clinical assessment by psychiatrist.
\end{abstract}

Results: Of 150 participants, majority were female $63.3 \%$. (95) and $36.7 \%$ (55) of the sample were between $26-45$ years. The prevalence of depressive disorder was 22\% (33).

The prevalence of depression was significantly associated with the recent diagnosis of epilepsy, use of multiple antiepileptic medications and duration of seizure free period $(p<0.05)$. There is a statistically significant association between prevalence of depression with the use of carbamazepine, topiramate, clobazam and phenobarbitone. Regression analysis revealed higher the duration individuals suffering from epilepsy were at lower odds of having depression compared with that of individuals suffering from lower duration of epilepsy. For each year in increase of duration of epilepsy, the odds of depression decreased by $2 \%(95 \% \mathrm{Cl} 0.3 \%$ to $5.1 \%)$.

Ceylon Medical Journal 2021; 66: 138-143

DOI: http://doi.org/10.4038/cmj.v66i3.9492
Conclusion: The prevalence of depression is high in patients with epilepsy. Risk of having depression is higher during the early phase of the illness. Therefore, it is important to screen patients with epilepsy for depressive disorder during the early course of the illness.

\section{Background}

Epilepsy is one of the commonest neurological conditions and about 50 million people are suffering from epilepsy worldwide [1]. The prevalence of the epilepsy in low- and middle-income countries is about twice that of high-income countries and $80 \%$ of the individuals live in developing countries. There are about 300000 individuals suffer from epilepsy in Sri Lanka with the crude prevalence is 9-11 per 1000 persons [2].

Epilepsy is also being regarded as a neurological condition with a wide range of neuropsychiatric manifestations [3]. The psychological conditions associated with individuals with epilepsy have been identified as one of the major concerns in the management of epilepsy [4]. Depression is reported to be a more important determinant of quality of life than seizure frequency in patients with epilepsy [5]. While suicide found to be 2.4 times higher in epileptic patients than the general population, it is 32 times higher in patients with epilepsy and comorbid depression [3]. Despite the importance of depression in epilepsy, it remains underrecognized and undertreated [6].

Depression has been reported as one of the most common psychiatric disorder in patients with epilepsy and the prevalence is ranged from $6.5 \%-49.5 \%[7,8]$. The wide range of prevalence suggests regional and cultural influences. Therefore, prevalence of depression and its associations among patients with epilepsy cannot be generalized or extrapolated between culturally and regional different settings. Many previous researches used screening tools to diagnose depressive disorder rather than detailed assessment by a clinician limiting the validity

${ }^{1}$ North Colombo Teaching Hospital, Ragama, Sri Lanka, ${ }^{2}$ Department of Psychiatry, Faculty of Medicine and Allied Sciences, Rajarata University, Sri Lanka, ${ }^{3}$ Department of Psychiatry, Faculty of Medicine, University of Kelaniya, Sri Lanka.

Correspondence: SSD, e-mail: <drsilumini@gmail.com>. Received 10 April 2021 and revised version 27 May 2021 accepted 15 June 2021

This is an open-access article distributed under the terms of the Creative Commons Attribution License, which permits unrestricted use, distribution, and reproduction in any medium, provided the original author and source are credited. 
of findings. No previous research from Sri Lanka has studied the prevalence and correlates of depression in patients with epilepsy. Therefore, aim of this study is to evaluate prevalence of depression and associated factors among individuals with epilepsy in Sri Lankan setting.

\section{Method}

The descriptive cross-sectional study was conducted at the epilepsy clinic of North Colombo Teaching hospital, Ragama, Sri Lanka. The Colombo North Teaching Hospital is the $3^{\text {rd }}$ largest tertiary care hospital in Sri Lanka with 1450 beds and is situated 18 kilometers north of capital, Colombo. Approximately 600 patients were registered in the epilepsy clinic. All the consenting adult patients with a diagnosis of epilepsy who were followed up at clinic between 01/8/2016 and 31/10/2016 were invited take part in the study. The patients who were unable to give consent and had impaired verbal expression were excluded from the study. Sample size was calculated to explore the prevalence of depression which was assumed to be $35 \%$ in Sri Lankan patients after studying similar studies with a probability of at least 95\%. Sample size was 143 and we recruited 150 patients.

Pre-tested, interviewer administered structured questionnaire was used to gather socio-demographic data and the information about associated factors were obtained from clinic records. Depressive disorder was initially screened with English and Sinhalese versions of validated Beck depression inventory II (BDI). The patients who were screened positive using a cutoff point $\geq 11$ was clinically assessed by a psychiatrist to confirm the diagnosis of depressive disorder according to the ICD 10 criteria. Beck depression inventory is a 21-item selfreporting scale which is widely used to diagnose depression. BDI has been used successfully to diagnose depression in patients with epilepsy [8-9]. BDI has been validated to use among Sinhalese speaking population in in Sri Lanka [10].

\section{Statistical analysis}

Descriptive statistics were computed to explain the socio-demographic characteristics, clinical variables, and prevalence of depression. Comparability of characteristics of patients with depression and without were calculated at the baseline analyzed by independent t-test for continuous variables and chi-square test for the categorical variables. Possible associated factors of depression in patients with epilepsy were initially analyzed by simple logistic regression model. Risk factors which were significant at $\mathrm{p}<0.05$ were considered for the final multiple logistic regression model for depression. Suitability of final model was assessed with Hosmer and Lemeshow goodness of fit test.

Ethical approval for the study was obtained from the Ethics Review Committee of the Faculty of Medicine, University of Kelaniya, Ragama. The collection and handling of data were carried out by primary author alone and anonymity of the subjects was ensured during discussion, thereby preserving the confidentiality.

Participants screened positive for depression, were referred to Psychiatry clinic North Colombo Teaching Hospital Ragama for appropriate treatment.

\section{Results}

Socio demographic data of 150 patients included in the study is summarized in Table 1. Most participants were females, married and had no employment or income. Clinical information of the participants is shown in Table 2. Most participants had tonic-clonic seizures and was treated with one antiepileptic.

Thirty-three participants (22\%) screened positive for depression with BDI II (Table 3). All of those 33 participants but one $(21.3 \%)$ was diagnosed to have depressive disorder by a psychiatrist using ICD 10 criteria. Of this, 4.6\% ( $\mathrm{n}=7)$ mildly, $8.0 \%(\mathrm{n}=12)$ moderately and $8.6 \%(\mathrm{n}=13)$ were severely depressed. Eighteen out of 33 patients with depression were undiagnosed and not treated before for depression.

There was a significant relationship between prevalence of depression and duration of seizure free period in patients with epilepsy $\left(\mathrm{X}^{2}=10.85, \mathrm{df}=5, \mathrm{p}=0.045\right)$. Depression was strongly associated with use of more than one antiepileptic medication $\left(\mathrm{X}^{2}=31.3, \mathrm{df}=3, \mathrm{p}=0.001\right)$ and greater the number of medications higher the risk $\left(X^{2}=25.8\right.$, $\mathrm{df}=3, \mathrm{p}=0.001)$ ). Furthermore, there was a statistically significant association between use of carbamazepine $\left(\mathrm{X}^{2}=18.8\right.$, $\left.\mathrm{df}=4, \mathrm{p}=0.01\right)$, topiramate $\left(\mathrm{X}^{2}=12.8, \mathrm{df}=3\right.$, $\mathrm{p}=0.045)$, clobazam $\left(\mathrm{X}^{2}=25.4, \mathrm{df}=3, \mathrm{p}=0.001\right)$, phenobarbitone. $\left(\mathrm{X}^{2}=4.124, \mathrm{df}=3, \mathrm{p}=0.0248\right)$ and depression. The prevalence of depression was increased with use of higher doses of topiramate, clobazam $(p<0.01)$. There was a significant association between prevalence of depression and past history of depression $\left(\mathrm{X}^{2}=40.6\right.$, $\mathrm{df}=1, \mathrm{p}=0.001)$. According to regression analysis, longer duration of epilepsy is associated with lower risk of developing depression. There was no statistically significant association with other factors. 
Table 1. Sociodemographic data of participants

\begin{tabular}{lll}
\hline & Characteristic & $\begin{array}{l}\text { Study population } \\
n=150\end{array}$ \\
\hline Sex & Male & $55(36.7 \%)$ \\
& Female & $95(63.3 \%)$ \\
Age & $18-25$ years & \\
& $26-45$ years & $26(17.3 \%)$ \\
& $46-65$ years & $55(36.7 \%)$ \\
Marital status & $>65$ years & $50(33.3 \%)$ \\
& & $19(12.7 \%)$ \\
Occupation & Married & $102(68 \%)$ \\
& Single & $47(31.3 \%)$ \\
& Divorced & $1(0.7 \%)$ \\
& & \\
& Employed & $60(40 \%)$ \\
& Unemployed & $90(60 \%)$ \\
& Yes & $69(46 \%)$ \\
& No & $81(54 \%)$ \\
& & \\
\hline
\end{tabular}

Table 2. Clinical data of participants

\begin{tabular}{lll}
\hline & Characteristic & $\begin{array}{l}\text { Study population } \\
n=150\end{array}$ \\
\hline Type of epilepsy & Tonic clonic seizures & $104(69.3 \%)$ \\
& Complex partial seizures & $15(10 \%)$ \\
& Simple partial seizures & $15(10 \%)$ \\
Myoclonic seizures & $5(3.3 \%)$ \\
Post traumatic seizures & $4(2.4 \%)$ \\
& \\
& $<5$ years & $37(24.7 \%)$ \\
$5-10$ years & $25(16.7 \%)$ \\
$11-15$ years & $20(13.3 \%)$ \\
$21-25$ years & $8(5.3 \%)$ \\
$26-30$ years & $19(12.7 \%)$ \\
$31-35$ years & $11(7.3 \%)$ \\
$36-40$ years & $7(4.7 \%)$ \\
$41-45$ years & $2(1.3 \%)$ \\
50 years & $5(3.3 \%)$ \\
\hline
\end{tabular}


Characteristic

Study population

$n=150$

Duration of seizure free period

$<1$ month

1-6 months

7 months- 1 year

2-5 years

6-10years

$>10$ years

Normal

No records

Generalized discharge

Left discharge

Right discharge

One

Two

Three

$>$ Three

Carbamazepine

Sodium Valproate

Clobazam

Phenytoin Sodium

Topiramate

Clonazepam

Phenobarbitone

Lamotrigine

Oxcarbazepine

Levetiracetam

Depression

Interictal psychosis

Other

Yes

No
$15(10 \%)$

$5(3.3 \%)$

$41(27.3 \%)$

$32(21.3 \%)$

$58(38.7 \%)$

13(8.7\%)

$1(0.7 \%)$

$77(51.3 \%)$

35(23.3\%)

$22(14.7 \%)$

$11(7.3 \%)$

5(3.3\%)

75(50\%)

$50(33.3 \%)$

19(12.7\%)

6(4\%)

$82(54.4 \%)$

$71(47.3 \%)$

$27(18 \%)$

$22(14.6 \%)$

$50(10.3 \%)$

$12(8 \%)$

$8(6.3 \%)$

$7(4.7 \%)$

$3(2 \%)$

$2(1.4 \%)$

$1(0.6 \%)$

$4(2.4 \%)$

$3(2 \%)$

$147(98 \%)$ 
Table 3. Distribution of severity of depression according to Beck Depression Inventory II

\begin{tabular}{lcc}
\hline BDI Score & Frequency & Percent \\
\hline Normal 0-10 & 117 & $80 \%$ \\
Mild11-16 & 4 & $3 \%$ \\
Borderline 17-20 & 11 & $5.7 \%$ \\
Moderate 21-30 & 14 & $8.7 \%$ \\
Severe 31-40 & 3 & $2 \%$ \\
Extreme 40 & 1 & $0.6 \%$ \\
Total & 150 & $100 \%$ \\
\hline
\end{tabular}

\section{Discussion}

Our study, first of its kind in Sri Lanka, confirms depression is indeed a common complication in patients with epilepsy as shown in similar studies worldwide. The prevalence found in our study is in line with pooled prevalence of depression in patients with epilepsy in Asia (25\%) and world (23.1\%) [11]. However, the prevalence in Sri Lanka is lesser than the prevalence reported in Ethiopia (45\% and 48\%), Gaza (63\%), Korea (62\%), Iraq (51.6\%), Nigeria (45\% and 85\%), Pakistan (60\%), Poland (49.2\%) (11-17).

This wide variation is possibly due to heterogenicity of the study design, cultural factors, population demographic and method of diagnosis of depression. It is interesting note that the study in our neighboring country Pakistan had a largely similar methodology but reported a much higher prevalence [12].

There are a multiple of circumstantial causes for depressive symptoms in patients with epilepsy such as seizure related injuries, stigma, limitation of engaging in certain activities, side effects of anti-epileptic medications and cultural beliefs about epilepsy. Whether these circumstantial causes alone can explain the higher prevalence of depression in patients with epilepsy is debated. Kanner suggested a possible bi-directional relationship between epilepsy and depression or the presence of common pathogenic mechanisms that facilitates the occurrence of one disorder in the presence of the other [8]. However, this bidirectional relationship is not supported by our finding of only $10 \%$ of participants reporting prior history of depression. However, the possibility of undiagnosed depression prior to onset of epilepsy should be considered.

Large number of studies showed that antiepileptic drug therapy increased the risk of depression in individuals with epilepsy and risk is high with polypharmacy [18-21] This is compatible with findings in our study. However, it should be noted that correlation between the number of antiepileptic drugs and the severity of depression may simply reflect the severity of epilepsy. Previous studies concluded that use of barbiturates, levetiracetam and topiramate is clearly associated with depression [22]. Our study also revealed a significant association between depression and use of carbamazepine, topiramate, clobazam and phenobarbitone.

Depression appeared to have strong negative affection of quality of life in people with epilepsy [23]. Our study revealed that risk of depression is high in early phase of epilepsy. Therefore, to improve the outcome, early recognition of depression is necessary. Educating medical staff in epilepsy clinics about symptoms of depression and introduction of screening tool to detect depression in routine practice are essential. Validated, easy to use screening tools such as Beck depression inventory II could be used to screen for depression in busy clinics as we did in our study. Patients who screen positive for depression should be referred to mental health services for further evaluation and management.

Future studies with larger sample size need to be conducted at national level to explore the impact of comorbid psychiatric illnesses among individuals with epilepsy.

\section{Limitations}

This study was conducted by using convenient sampling method. Therefore, sample does not represent the general population in Sri Lanka and generalization of the results of this study may be limited. Inadequate sample size is another limitation and may have influenced in detecting associations between prevalence of depression and certain demographic and clinical factors. Severity of epilepsy is a confounding factor that has an effect on correlation between number of antiepileptic drugs and the severity of depression. 


\section{Authors' contributions}

SS has conducted research work, data collection and prepared the manuscript. AI has done the data analysis and contributed intellectual input in protocol and manuscript writing. KALAK and AR provided intellectual input in protocol and manuscript writing, and supervision of the article. All the authors have seen and approved the final version of the article.

\section{Conflicts of Interest}

None declared.

\section{Acknowledgements}

The authors gratefully acknowledge the support provided by Director, Consultant neurologist, medical officers, other staff members and patients of epilepsy clinic at North Colombo Teaching Hospital.

\section{Ethics approval}

Ethics approval was obtained from the Ethics Review Committee of the Faculty of Medicine, University of Kelaniya.

\section{Source of funding}

This research did not receive any specific fundings from funding agencies

\section{References}

1. Fiest KM, Sauro KM, Wiebe S, et al. Prevalence and incidence of epilepsy: A systematic review and metaanalysis of international studies. Neurology 2017; 17: 83(3) 296-303.

2. Merlis JK. Proposal for an international classification of epilepsies. Epilepsia 1970; 11(1): 114-9.

3. Agrawal N, Govender S, Epilepsy and Neuropsychiatric co morbidities. Adv Psychiatr Treat 2011; 17(1) 44-53.

4. World Health Organization (WHO). Epilepsy in the WHO Africa Region: Bridging the Gap.WHO: Geneva, 2004.

5. Boylan LS, Flint LA, Labovitz DL, et al. Depression but not seizure frequency predicts quality of life in treatmentresistant epilepsy. Neurology 2004; 62: 258-61.

6. Wiegartz P, Seidenberg M, Woodard A, Gidal B, Hermann B. Co morbid psychiatric disorder in chronic epilepsy: recognition and etiology of depression. Neurology 1999; 53(suppl 2): S3-S8.

7. Fiest KM, Dykeman J, Patten SB, et al. Depression in epilepsy: a systematic review and meta-analysis. Neurology 2013; 80(6): 590-9.

8. Dessie G, Mulugeta H, Leshargie CT, Wagnew F, Burrowes $\mathrm{S}$. Depression among epileptic patients and its association with drug therapy in sub-Saharan Africa: A systematic review and meta-analysis. PLoS One 2019; 14(3): e0202613.

9. Kanner AM. Depression in epilepsy: prevalence, clinical semiology, pathogenic mechanism and treatment. Biol Psychiatry 2003; 54(3): 388-98.

10. Rodrigo A, Kuruppuarachchi KALA, Pathmeswaran A. Validation of Beck Depression Inventory II among Sinhalese speaking population in Sri Lanka. Sri Lanka J. Psychiatry 2015; 6(2): 20-24.

11. Abu Sheer A. Depression among Epileptic Patients in Governmental Community Mental Health Centers in Gaza Strip. Oxford: The Islamic University-Gaza; Gaza City, 2012.

12. Yousafzai AU, Yousafzai AW, Taj R. Frequency of depression in epilepsy: a hospital-based study. J Ayub Med Coll Abbottabad 2009; 21(2): 73-5.

13. Asadi-Pooya AA, Sperling MR. Depression and anxiety in patients with epilepsy, with or without other chronic disorders. Iran Red Crescent Med J 2011; 13(2): 112-6.

14. Lee SA, Lee SM, No YJ. Factors contributing to depression in patients with epilepsy. Epilepsia 2010; 51(7): 1305-8.

15. Onwuekwe IO, Ekenze OS, Bzeala-Adikaibe O, Ejekwu JU. Depression in patients with epilepsy: A study from Enugu, South East Nigeria. Ann Med Health Sci Res. 2012; 2(1): 10-13.

16. Bifftu BB, Dachew BA, Tiruneh BT, Tebeje NB. Depression among people with epilepsy in Northwest Ethiopia: a cross-sectional institution-based study. BMC Res Notes 2015; 8: 585.

17. Grabowska-Grzyb A, Jedrzejczak J, Naganska E, Fiszer U. Risk factors for depression in patients with epilepsy. Epilepsy Behav 2006; 8(2): 411-17.

18. Mula M, Sander JW. Negative effects of antiepileptic drugs on mood in patients with epilepsy. Drug Saf 2007; 30(7): 555-67.

19. Ridsdale L, Ashworth M, Richardson MP, Gulliford MC. Epilepsy mortality and risk factors for death in epilepsy: a population-based study. Br J Gen Pract 2011; 61(586): e271-8.

20. Kiko N. Prevalence and factors associated with depression among patients with epilepsy in a Kenyan tertiary care hospital. Aga Khan Hospital: Nairobi, 2012.

21. Tegegne MT, Mossie TB, Awoke AA, Assaye AM, Gebrie BT, Eshetu DA. Depression and anxiety disorders among epileptic people at Amanuel Specialized Mental Hospital, Addis Ababa, Ethiopia. BMC psychiatry 2015; 15: 210.

22. Mula M, Trimble MR, Sander JW. Are psychiatric adverse events of antiepileptic drugs a unique entity? A study on topiramate and levetiracetam. Epilepsia 2007; 48(12): 2322-6.

23. Ball HA, Siribaddana SH, Kovas Y, et al. Epidemiology and symptomatology of depression in Sri Lanka: A cross sectional population-based survey in Colombo District. $J$ Affect Disord 2010; 123(1-3): 188-96. 